Pacific Journal of Mathematics

HYPERFINITE NON NEUMANN ALGEBRAS AND POISSON
BOUNDARIES OF TIME DEPENDENT RANDOM WALKS 


\title{
HYPERFINITE VON NEUMANN ALGEBRAS AND POISSON BOUNDARIES OF TIME DEPENDENT RANDOM WALKS
}

\author{
A. Connes AND E. J. Woods \\ Dedicated to the memory of Henry A. Dye
}

\begin{abstract}
We consider the problem of characterizing Poisson boundaries of group-invariant time-dependent Markov random walks on locally compact groups $G$. We show that such Poisson boundaries, which by construction are naturally $G$-spaces, are amenable and approximately transitive (see Definition 1.1 and Theorem 2.2).

We also establish a relationship between von Neumann algebras and Poisson boundaries when $G=R$ or $Z$. More precisely, there is naturally associated to an eigenvalue list for an ITPFI factor $M$, a group-invariant time-dependent Markov random walk on $R$ whose Poisson boundary is the flow of weights for $M$ (Theorem 3.1).
\end{abstract}

0. Introduction. 'Henry Dye's work has a lasting impact on ergodic theory and operator algebras. We present this paper, which deals with both of these subjects, as a tribute to his mathematical achievements and his gentle and unassuming character.

We consider the problem of characterizing Poisson boundaries of group-invariant time-dependent Markov random walks on locally compact groups $G$. We show that such Poisson boundaries, which by construction are naturally $G$-spaces, are amenable and approximately transitive (see Definition 1.1 and Theorem 2.2). We believe that the converse also holds, namely that these two conditions precisely characterize such Poisson boundaries. Under the pressure of time, we have not yet completed our proof. However it is true in the transitive case (Theorem 2.4), and when $G=R$ or $Z$ (Theorems 3.2 and 3.4). Theorem 2.6 is the beginning of our attack on the general case.

We also establish a relationship between von Neumann algebras and Poisson boundaries when $G=R$ or $Z$. More precisely, there is naturally associated to an eigenvalue list for an ITPFI factor $M$, a group-invariant time-dependent Markov random walk on $R$ whose Poisson boundary is the flow of weights for $M$ (Theorem 3.1). Theorem 3.3 gives the corresponding result for $G=Z$. This unexpected identification has interesting applications in both directions. Using 
some non-trivial theorems from operator algebras, we determine the Poisson boundaries for $G=R$ and $Z$ (Theorems 3.2 and 3.4). Conversely, the harmonic function description of Poisson boundaries gives a remarkably simple proof of the $T$-set condition for ITPFI factors (Theorem 4.2). Just as the ITPFI factors are related to the Poisson boundaries of random walks on $R$ and $Z$, the hyperfinite factors (which properly contain the ITPFI factors) are related to the Poisson boundaries of matrix-valued random walks on $R$ and $Z$ (see $\S 3$ (iii)).

Section 1 discusses the relevant results from von Neumann algebras, including the approximate transitivity condition. In $\S 2$ we recall the construction of the Poisson boundary, and present our incomplete characterization. In $\S 3$ we give the relationships between approximately type I (hyperfinite) factors and random walks on $R$ and $Z$. Section 4 contains an application to the $T$ set. Appendix A identifies the Poisson boundary as the Mackey range of a certain cocycle. Appendix B contains a remark on the Choquet boundary.

Notation. All groups are second countable locally compact. Groupinvariant Markov random walk always means a right group-invariant Markov random walk (see §2). Haar measure is always left Haar measure. All measure spaces are standard measure spaces. If $\mu$ is a measure, then $\|\mu\|$ always denotes the $L^{1}$-norm of $\mu$. If $X$ is a $G$ space, $\mu$ is a finite measure on $X$, and $\lambda$ is a finite measure on $G$, then $\lambda * \mu=\int d \lambda(g) g^{\mu}(x)$ where $d g^{\mu}(x)=d \mu\left(g^{-1} x\right) . \tilde{\lambda}$ is the measure defined by $d \tilde{\lambda}(g)=d \lambda\left(g^{-1}\right)$. If $A$ is a subset of a convex $G$-space, then $\overline{\mathrm{Co}}_{G} A$ denotes the closed convex hull generated by all $\alpha_{g}(a), a \in A$, $g \in G$.

1. Eigenvalue lists, ITPFI factors, and the flow of weights. A factor which can be constructed as an infinite tensor product of finite type I factors is called an ITPFI factor. These factors are the noncommutative analogues of product measures of probability on finite spaces. An ITPFI factor $M$ is determined by giving an eigenvalue list $\left(\lambda_{n 1}, \ldots, \lambda_{n k_{n}}\right)_{n \in N}$ where $\lambda_{n 1} \geq \lambda_{n 2} \geq \cdots \geq \lambda_{n k_{n}}>0$ and $\sum_{j=1}^{k_{n}} \lambda_{n j}=1$ for all $n . M$ is then of the form $M=\bigotimes_{n=1}^{\infty}\left(M_{n}, \varphi_{n}\right)$ on the Hilbert space $H=\bigotimes_{n=1}^{\infty}\left(H_{n}, \varphi_{n}\right)$ where the $M_{n}$ are type $\mathrm{I}_{k_{n}}$ factors acting on $H_{n}$, and $\phi_{n}(x)=\left(x \varphi_{n}, \varphi_{n}\right)$ is a faithful state on $M_{n}$. The $\lambda_{n j}$ are the eigenvalues of $\rho_{n} \in M_{n}$ where $\phi_{n}(x)=$ Trace $\rho_{n} x$.

The ITPFI factors belong to a larger family, namely the approximately type I factors. A von Neumann algebra $M$ is said to be approximately type I (or hyperfinite) if it is of the form $M=\left(\bigcup_{n=1}^{\infty} M_{n}\right)^{\prime \prime}$ 
where $M_{n} \subset M_{n+1}$ and each $M_{n}$ is a finite-dimensional matrix algebra. In the classification problem the approximately type I factors are the most natural class [4]. The type $\mathrm{II}_{1}$ approximately type $\mathrm{I}$ factor has long been known to be unique [13]. The remaining non-type I approximately type I factors are completely characterized by their flow of weights (an ergodic $R$-action which is naturally defined as an invariant of the factor) $[12,4,6,10]$. All ergodic $R$-actions appear in this way. It is trivial that ITPFI implies approximately type I, but the converse is false and non-trivial [11, 3]. Recently we have completely characterized the ITPFI factors by a new ergodic property of their flow of weights, which we call approximate transitivity [7].

Definition 1.1 Let $G$ be a Borel group, $(X, \nu)$ a standard measure space, and $\alpha: G \rightarrow \operatorname{Aut}(X, \nu)$ a Borel homomorphism. We say that the action is approximately transitive if given $n<\infty$, finite measures $\mu_{1}, \ldots, \mu_{n}<\nu$, and $\varepsilon>0$, there exists a finite measure $\mu<\nu$, $g_{1}, \ldots, g_{m} \in G$ for some $m<\infty$, and $\lambda_{j k} \geq 0, k=1, \ldots, m$, such that

$$
\left\|\mu_{j}-\sum_{k=1}^{m} \lambda_{j k} \alpha_{g_{k}} \mu\right\| \leq \varepsilon, \quad j=1, \ldots, n .
$$

If $G=Z$ and $\alpha$ is approximately transitive, then we say that $T=\alpha(1)$ is approximately transitive.

For ITPFI factors, there is a straightforward formula for the flow of weights in terms of the eigenvalue list (see $\S 3$ ). But since this formula involves an ergodic decomposition, there is the obvious question of giving a simple interpretation of the flow in terms of the eigenvalue list. In this paper we give an answer to this question by identifying the flow of weights for an ITPFI factor $M$ as the Poisson boundary of a groupinvariant Markov random walk on $R$ which is naturally associated to an eigenvalue list for $M$. The Poisson formula then identifies the flow of weights with the harmonic functions of the random walk.

2. Group-invariant Markov random walks, approximate transitivity, and amenability. Recall that a right group-invariant Markov random walk on a group $G$ is determined by a sequence of probability measures $\sigma_{n}$ ([14], p. 27). Namely the transition probability from $g \in G$ to the Borel set $A$, at the $n$th step, is given by $P_{n}(g, A)=\sigma_{n}\left(g^{-1} A\right)$. In particular $P_{n}(g, g A)=P_{n}(e, A)=\sigma_{n}(A)$. The right random walk is invariant under left translations (see (2.1) below). One defines a Markov chain of random variables $Y_{n}$ as follows. Let $X_{n}\left(\left(g_{k}\right)\right)=g_{n}$ 
be the canonical sequence of $G$-valued independent random variables with distributions $\sigma_{n}$ on the probability space $\left(G^{N}, \Pi_{n=1}^{\infty} \sigma_{n}\right)$. Let $\sigma_{0}$ be a probability distribution equivalent to Haar measure on $G$ ( $\sigma_{0}$ is the initial distribution for the random walk). Then on the probability space $\Omega=\left(G \times G^{N}, \sigma_{0} \times \sigma\right)$ we define $Y_{0}\left(\left(g_{k}\right)\right)=g_{0}$ and $Y_{n+1}=$ $Y_{n} X_{n+1}, n=0,1,2, \ldots$. Note that $Y_{n}$ has the distribution $\sigma_{0} * \sigma_{1} *$ $\cdots * \sigma_{n}$ which evolves, at the $n$th step, from the initial distribution $\sigma_{0}$ according to the random walk. $G$ acts on $\Omega$ by

$$
g\left(g_{n}\right)=\left(g g_{n}\right), \quad n=0,1,2, \ldots .
$$

In general we shall shift freely from left group actions on a measure space $\Omega$ to right group actions through the equality $g x=x g^{-1}$. The asymptotic algebra of the random walk is defined by $\mathscr{A}_{\Omega}=$ $\bigcap_{n=1}^{\infty} \sigma\left(Y_{k}, k \geq n\right)$ where $\sigma\left(Y_{k}, k \geq n\right)$ denotes the abelian von Neumann subalgebra of $L^{\infty}(\Omega)$ consisting of functions measurable with respect to the $\sigma$-algebra generated by the $Y_{k}, k \geq n$. Thus an asymptotic random variable is one which does not depend on the first $n$ coordinates of the path. The Poisson boundary $(B, \mu)$ of the random walk is defined in terms of the asymptotic algebra by $\mathscr{A}_{\Omega}=L^{\infty}(B, \mu)$. The Poisson boundary is a $G$-space under the restriction of the $G$ action on $L^{\infty}(\Omega)$ to $\mathscr{A}_{\Omega}$ (which is invariant under this action). In fact, the Poisson boundary can be identified as the Mackey range of a certain cocycle $\alpha:\left(G \times G^{N}\right) \times K \rightarrow G$ where $K$ is the restricted infinite product of copies of $G$ (see appendix A).

We define a harmonic function $h$ for the random walk to be a sequence $h_{n} \in L^{\infty}(G, d g)$ satisfying

$$
h_{n}(g)=\int h_{n+1}\left(g g^{\prime}\right) d \sigma_{n+1}\left(g^{\prime}\right)=h_{n+1} * \tilde{\sigma}_{n+1}
$$

where $\tilde{\sigma}$ denotes the image of $\sigma$ under the map $g \rightarrow g^{-1}$. This condition is just the meanvalue property, namely $h_{n}(g)$ is the average, according to the random walk, of $h_{n+1}$. (This definition corresponds to the $Q$-harmonic functions in [14].) The harmonic function $h$ is called bounded if $\sup _{n}\left\|h_{n}\right\|_{\infty}<\infty$. The Poisson formula (see [14], Proposition 2.3, p. 165)

$$
h_{n}(g)=\int \mathscr{Z}\left(g_{0}, g_{1}, \ldots, g_{n} \ldots\right) d P_{g}^{(n)}\left(g_{n}, g_{n+1}, \ldots\right)
$$

associates a bounded harmonic function to any bounded asymptotic random variable $\mathscr{Z} . P_{g}^{(n)}$ is the Markov measure for the Markov random walk which begins at the $r$ th step at the point $g$ and then continues according to the random walk defined by the sequence $\sigma_{n+1}$, 
$\sigma_{n+2}, \ldots$. Note that $\mathscr{Z}$ does not depend on the first $n$ coordinates. This association is one-to-one since one can use the martingale theorem to show that

$$
\mathscr{Z}(\omega)=\lim _{n \rightarrow \infty} h_{n}\left(Y_{n}(\omega)\right)
$$

for a.e. $\omega \in \Omega$. The operators $P_{k, n}: L^{\infty}(G, d g) \rightarrow L^{\infty}(G, d g)$ defined by

$$
P_{k, n} f=f * \tilde{\sigma}_{n} * \tilde{\sigma}_{n-1} * \cdots * \tilde{\sigma}_{k+1}, \quad k<n,
$$

satisfy

$$
h_{k}=P_{k, n} h_{n}, \quad k<n .
$$

This identification of $L^{\infty}(B, \mu)$ with bounded harmonic functions is basic for our results. We begin with

LEMMA 2.1. Let $\varphi \in L^{1}(G), h=\left(h_{n}\right) \in L^{\infty}(B)$. Then the equation

$$
\left\langle h, \Pi_{n} \varphi\right\rangle=\int_{G} h_{n}(g) \bar{\varphi}(g) d g
$$

defines a contraction $\Pi_{n}: L^{1}(G) \rightarrow L^{1}(B)$ satisfying

$$
\Pi_{n}\left(P_{k, n}^{*} \varphi\right)=\Pi_{k}(\varphi), \quad k<n .
$$

Proof. For fixed $\varphi$, the right-hand side of (2.6) is a normal linear functional on $L^{\infty}(B)$. Therefore it defines an element $\Pi_{n} \varphi$ of the predual $L^{\infty}(B)_{*}=L^{1}(B)$. It follows directly from (2.6) that $\left\|\Pi_{n}\right\| \leq 1$. (2.7) follows from

$$
\left\langle\Pi_{k} \varphi, h\right\rangle=\left\langle\varphi, h_{k}\right\rangle=\left\langle\varphi, P_{k, n} h_{n}\right\rangle=\left\langle P_{k, n}^{*} \varphi, h_{n}\right\rangle .
$$

THEOREM 2.2. The Poisson boundary of a group-invariant Markov random walk on the group $G$, considered as a G-space, is approximately transitive and amenable.

Proof. (i) Since approximate transitivity (see Definition 1.1) is an approximation property in $L^{1}(B)$, it suffices to prove it on a dense set. We will do this by exhibiting $L^{1}(B, \mu)$ as an inductive limit of $L^{1}(G, d g)$, i.e. of transitive actions of $G$ on $G$. (Transitive actions are trivially approximately transitive.)

Let $h \in L^{\infty}(B), \varphi \in L^{1}(G)$. Since $h \neq h^{\prime}$ implies $h_{n} \neq h_{n}^{\prime}$ for some $n$, the linear functionals $L_{n, \varphi}(h)=\left\langle h, \Pi_{n} \varphi\right\rangle=\left\langle h_{n}, \varphi\right\rangle$ separate points in $L^{\infty}(B)$. The Hahn-Banach theorem now implies that 
$\bigcup_{n \in N} \Pi_{n}\left(L^{1}(G)\right)$ is dense in $L^{1}(B)$. It follows from (2.7) that $\Pi_{n}\left(L^{1}(G)\right) \subset \Pi_{n+1}\left(L^{1}(G)\right)$, so that the embedding is inductive.

(ii) The idea of the proof of amenability is that $L^{\infty}(B)$ is a projective limit of $L^{\infty}(G)$, and that the transitive action of $G$ on $G$ is amenable. Our definition of amenability $([\mathbf{1 5}],[8])$ is the existence of a map $P: L^{\infty}(B \times G) \rightarrow L^{\infty}(B)$ satisfying

(i) $P \geq 0$,

(ii) $P(F(b))=F(b)$, and

(iii) $P(h F)=h(P F)$ for all $h \in G$,

where $h F(b, g)=F\left(b h, h^{-1} g\right)$ and $h F(b)=F(b h)$. We begin by proving the amenability of the transitive action of $G$ on $G$. Let $F \in$ $L^{\infty}(G \times G)$ and let $\rho$ be any probability measure on $G$. Then

$$
\left(P_{\rho} F\right)\left(g_{1}\right)=\int_{g_{2} \in G} F\left(g_{1}, g_{2}\right) d \rho\left(g_{2}^{-1} g_{1}\right)
$$

satisfies (i) and (ii) trivially, and

$$
\begin{aligned}
h\left(P_{\rho} F\right) & =\int_{g_{2}^{\prime} \in G} F\left(h^{-1} g_{1}, g_{2}^{\prime}\right) d \rho\left(\left(g_{2}^{\prime}\right)^{-1} h^{-1} g_{1}\right) \\
& =\int_{g_{2} \in G} F\left(h^{-1} g_{1}, h^{-1} g_{2}\right) d \rho\left(g_{2}^{-1} g_{1}\right)=P_{\rho}(h F)
\end{aligned}
$$

where we set $g_{2}=h g_{2}^{\prime}$. Note that with our conventions, when $B=G$, we have $(g) h=\left(h^{-1} g\right)$.

Now let $F \in L^{\infty}(B \times G)$. For fixed $g_{2}, F\left(b, g_{2}\right) \in L^{\infty}(B)$ for a.e. $g_{2} \in G$. Hence $F\left(b, g_{2}\right)$ corresponds to harmonic functions $F_{n}\left(g_{1}, g_{2}\right)$ satisfying $P_{k, n} F_{n}\left(g_{1}, g_{2}\right)=F_{k}\left(g_{1}, g_{2}\right)$ where $k<n$ and $P_{k . n}$ acts on the first variable $g_{1}$. Note that $P F$ is determined by giving $\langle P F, \varphi\rangle$ on the dense set of $\varphi \in L^{1}(B)$, which, for some $k<\infty$, are of the form $\varphi=\Pi_{k}\left(\varphi_{k}\right), \varphi_{k} \in L^{1}(G)$. Now let $\left(\rho_{n}\right)_{n \in N}$ be probability measures on $G$, and let $\omega$ be an ultrafilter on $N$. We define

$$
\langle P F, \varphi\rangle=\lim _{\omega} \int_{G}\left(P_{\rho_{n}} F_{n}\right)\left(g_{1}\right)\left(\overline{P_{k, n}^{*} \varphi_{k}}\right)\left(g_{1}\right) d g_{1}
$$

where $P_{\rho_{n}}$ is given by (2.8). Trivially $P \geq 0$ so (i) is satisfied. Since $P(1)=1$, it follows that $\|P F\|_{\infty} \leq\|F\|_{\infty}$ and hence $P F$ is well defined by $(2.10)$. If $F\left(b, g_{2}\right)=F(b)$ has no $g_{2}$ dependence, then $F_{n}\left(g_{1}, g_{2}\right)=$ $F_{n}\left(g_{1}\right)$ and $P_{\rho_{n}} F_{n}=F_{n}$. Using (2.5), (2.6) and (2.10) we have

$$
\langle P F, \varphi\rangle=\lim _{\omega}\left\langle P_{k, n} F_{n}, \varphi_{k}\right\rangle=\lim _{\omega}\left\langle F_{k}, \varphi_{k}\right\rangle=\langle F, \varphi\rangle .
$$

Hence (ii) is satisfied. 
To check condition (iii), note that using (2.1) we have for $G \in$ $L^{\infty}(B)$,

$$
\begin{aligned}
\left\langle h G, \Pi_{k}\left(\varphi_{k}\right)\right\rangle & =\left\langle(h G)_{k}, \varphi_{k}\right\rangle \\
& =\int G_{k}\left(h^{-1} g\right) \bar{\varphi}_{k}(g) d g \\
& =\int G_{k}(g) \bar{\varphi}_{k}(h g) d g=\left\langle G_{k}, h^{-1} \varphi_{k}\right\rangle .
\end{aligned}
$$

Hence

$$
h^{*}\left(\Pi_{k}\left(\varphi_{k}\right)\right)=\Pi_{k}\left(h^{-1} \varphi_{k}\right)
$$

Thus

$$
\langle h(P F), \varphi\rangle=\left\langle P F, h^{*} \varphi\right\rangle=\lim _{\omega}\left\langle P_{\rho_{n}} F_{n}, P_{k, n}^{*}\left(h^{-1} \varphi_{k}\right)\right\rangle .
$$

Using (2.9) and (2.10) we have

$$
\begin{aligned}
\langle P(h F), \varphi\rangle & =\lim _{\omega}\left\langle P_{\rho_{n}}\left(h F_{n}\right), P_{k, n}^{*} \varphi_{k}\right\rangle \\
& =\lim _{\omega}\left\langle h\left(P_{\rho_{n}} F_{n}\right), P_{k, n}^{*} \varphi_{k}\right\rangle \\
& =\lim _{\omega}\left\langle P_{\rho_{n}} F_{n}, h^{-1}\left(P_{k, n}^{*} \varphi_{k}\right)\right\rangle \\
& =\lim _{\omega}\left\langle P_{\rho_{n}} F_{n}, P_{k, n}^{*}\left(h^{-1} \varphi_{k}\right)\right\rangle
\end{aligned}
$$

where the last step used the fact that the right-invariant Markov random walk commutes with left translations. Thus (iii) is satisfied.

Zimmer has proven the amenability of the Poisson boundary of stationary group-invariant Markov random walks ([15], Theorem 5.2). However his mehod does not seem adaptable to the non-stationary case.

REMARK 2.3. Theorem 2.2 has an obvious generalization to the case where the state space for the random walk at the $n$th step is a $G$-space $X_{n}$, rather than the group $G$ itself. Group-invariance has the obvious meaning here, but the transition probability at each step is no longer specified by giving a single probability measure on $G$. It is clear from the proof of Theorem 2.2 that if each $X_{n}$ is approximately transitive (resp. amenable) then the Poisson boundary is approximately transitive (resp. amenable). In particular, for matrix-valued random walks on $G$ (see $\S 3$ (iii) for a discussion of matrix-valued random walks on $R$ and $Z$ ), the state space $X_{n}$ is a finite disjoint union of copies of $G$ acting transitively on $G$, and hence amenable. It follows that the Poisson boundary of a matrix-valued random walk on $G$ is an amenable $G$-space. 
Theorem 2.4. A transitive amenable action of a group $G$ is the Poisson boundary of a group-invariant Markov random walk on $G$.

We prove first

LeMma 2.5. Let $\mathscr{R}_{j}, j=1,2$, be group-invariant Markov random walks on $G$ defined by the probability measures $\left(\sigma_{k}^{(j)}\right)_{k \in N}$ on $G$. If (2.12) $\lim _{n, m \rightarrow \infty}\left\|\sigma_{n}^{(1)} * \sigma_{n+1}^{(1)} * \cdots * \sigma_{n+m}^{(1)}-\sigma_{n}^{(2)} * \sigma_{n+1}^{(2)} * \cdots * \sigma_{n+m}^{(2)}\right\|=0$

then the Poisson boundaries of $\mathscr{R}_{1}$ and $\mathscr{R}_{2}$ are isomorphic (i.e. they are conjugate as $G$-spaces).

Proof. Let $\left(B_{j}, \mu_{j}\right)$ denote the Poisson boundaries of $\mathscr{R}_{j}$. Recall that any $h^{j} \in L^{\infty}\left(B_{j}, \mu_{j}\right)$ corresponds to a harmonic function $\left(h_{n}^{j}\right)_{n \in N}$, $h_{n}^{j} \in L^{\infty}(G, d g)$. On $L^{\infty}(G, d g)$ we define the operators

$$
P_{n, m}^{j} f=f * \tilde{\sigma}_{m}^{(j)} * \tilde{\sigma}_{m-1}^{(j)} * \cdots * \tilde{\sigma}_{n+1}^{(j)}, \quad n<m
$$

$($ see $(2.4))$. Then we have $\left\|P_{n, m}^{j}\right\| \leq 1, P_{n, m}^{j} h_{m}^{j}=h_{n}^{j}$, and (2.12) becomes

$$
\lim _{n, m \rightarrow \infty}\left\|P_{n, m}^{1}-P_{n, m}^{2}\right\|=0 .
$$

We will show that the equation

$$
h_{n}^{2}=\lim _{m \rightarrow \infty} P_{n, m}^{2} h_{m}^{1}
$$

defines an isometry $\Theta$ from $L^{\infty}\left(B_{1}, \mu_{1}\right)$ onto $L^{\infty}\left(B_{2}, \mu_{2}\right)$. Let $\left(h_{n}^{1}\right)$ be harmonic for $\mathscr{R}_{1}$. Define $h_{n, m}^{2}=P_{n, m}^{2} h_{m}^{1}, m>n$. If $m^{\prime}>m$ we have

$$
h_{n, m^{\prime}}^{2}-h_{n, m}^{2}=P_{n, m}^{2}\left[P_{m, m^{\prime}}^{2}-P_{m, m^{\prime}}^{1}\right] h_{m^{\prime}}^{1} \text {. }
$$

Since $\left\|h^{1}\right\|_{\infty}=\sup \left\|h_{n}^{1}\right\|_{\infty}$, it follows from (2.14) that $h_{n, m}^{2}$ is Cauchy in $m$, and hence the limit in (2.15) exists in $L^{\infty}$-norm. In particular we have

$$
\sup _{n}\left\|h_{n}^{2}\right\|_{\infty} \leq\left\|h^{1}\right\|_{\infty}
$$

and hence $\|\Theta\| \leq 1$. We also have

$$
h_{n}^{2}=\lim _{m \rightarrow \infty} P_{n, n+1}^{2} P_{n+1, m}^{2} h_{m}^{1} \text {. }
$$

Since $\left\|P_{n, n+1}^{2}\right\| \leq 1$ and $\left\|h_{n+1}^{2}-P_{n+1, m}^{2} h_{m}^{1}\right\| \underset{m \rightarrow \infty}{\rightarrow} 0$ we have

$$
h_{n}^{2}=P_{n, n+1}^{2} h_{n+1}^{2} \text {, }
$$


i.e. $\left(h_{n}^{2}\right)$ is harmonic for $\mathscr{R}_{2}$. Thus we have

$$
\Theta: L^{\infty}\left(B_{1}, \mu_{1}\right) \rightarrow L^{\infty}\left(B_{2}, \mu_{2}\right) .
$$

To prove that $\Theta$ is onto, we define $\Psi: L^{\infty}\left(B_{2}, \mu_{2}\right) \rightarrow L^{\infty}\left(B_{1}, \mu_{1}\right)$ by

$$
\left(\Psi h^{2}\right)_{n}=\lim _{m \rightarrow \infty} P_{n, m}^{1} h_{m}^{2}
$$

Using (2.14) we have

$$
\begin{aligned}
\left(\Theta\left(\Psi h^{2}\right)\right) & =\lim _{m \rightarrow \infty} P_{n, m}^{2}\left(\Psi h^{2}\right)_{m} \\
& =\lim _{m, m^{\prime} \rightarrow \infty} P_{n, m}^{2} P_{m, m^{\prime}}^{1} P_{m^{\prime}}^{2} \\
& =\lim _{m, m^{\prime} \rightarrow \infty}\left\{h_{n}^{2}+P_{n, m}^{2}\left[P_{m, m^{\prime}}^{1}-P_{m, m^{\prime}}^{2}\right] h_{m^{\prime}}^{2}\right\} \\
& =h_{n}^{2},
\end{aligned}
$$

which proves that $\Theta \Psi=1$, and hence $\Theta$ is onto. Since $\|\Psi\| \leq 1$, it follows that $\Theta$ is an isometry. Finally, since $P_{n, m}^{2}$ commutes with left translations (see (2.1)), it follows directly from (2.15) that $\Theta$ intertwines the $G$-actions.

Proof of Theorem 2.4. A transitive action of $G$ is necessarily the canonical action of $G$ on $G / H$ where $H$ is the fixed-point subgroup ( $G / H$ denotes the space of right cosets). This action is amenable if and only if $H$ is amenable ([16], Proposition 4.3.2). Since $G$ and therefore $H$ is second countable, Reiter's condition ([9], pp. 43-44) gives a sequence of probability measures $\mu_{n}$ on $H$ such that $\left\|g \mu_{n}-\mu_{n}\right\|_{1} \rightarrow 0$ for all $g \in H$, where $\left(g \mu_{n}\right)(A)=\mu_{n}\left(g^{-1} A\right)$. We will show that for the random walk $\mathscr{R}_{1}$ defined by the $\mu_{n}$, the harmonic functions are precisely the constant sequences $h_{n}(g)=f(\Pi g)$ where $f \in L^{\infty}(G / H)$ and $\Pi: G \rightarrow G / H$ is the quotient map. I.e. the Poisson boundary is $L^{\infty}(G / H)$.

Let $f \in L^{\infty}(G / H)$. Since the measures $\mu_{n}$ are supported on $H$ and $\Pi\left(g g^{\prime}\right)=\Pi(g)$ if $g^{\prime} \in H$, it follows from (2.2) that $h_{n}(g)=f(\Pi(g))$ is harmonic. Conversely, let $\left(h_{n}\right)$ be harmonic. Fix $x \in H$. Then for all $g \in G$ we have, using (2.2),

$$
\begin{aligned}
\mid h_{m}(g) & -h_{m}(g x) \mid \\
= & \left|\int h_{m+1}\left(g g^{\prime}\right)\left[d \mu_{m+1}\left(g^{\prime}\right)-d \mu_{m+1}\left(x^{-1} g^{\prime}\right)\right]\right| \\
\leq & \|h\|_{\infty}\left\|\mu_{m+1}-x \mu_{m+1}\right\| \underset{m \rightarrow \infty}{\rightarrow} 0 .
\end{aligned}
$$


Hence

$$
\begin{aligned}
\left|h_{n}(g)-h_{n}(g x)\right| & =\left|\left(P_{n, m} h_{m}\right)(g)-\left(P_{n, m} h_{m}\right)(g x)\right| \\
& \leq \mid h_{m}(\cdot)-h_{m}(\cdot x) \|_{\infty} \underset{m \rightarrow \infty}{\rightarrow} 0
\end{aligned}
$$

and $h_{n}(g)$ is constant on $H$ cosets for any $n$. It now follows from (2.2) that $h_{m}(g)=h_{n}(g)$ for all $m<n$, and hence $h_{n}(g)=f(\Pi(g))$ for some $f \in L^{\infty}(G / H)$.

The random walk $\mathscr{R}_{1}$ is confined to the subgroup $H$. The desired random walk $\mathscr{R}_{2}$ is now defined by the measures $\sigma_{n}=\alpha_{n} \mu_{n}+\left(1-\alpha_{n}\right) \zeta_{n}$ where the $\zeta_{n}$ are probability measures equivalent to Haar measure on $G, 0<\alpha_{n}<1$, and the $\alpha_{n} \rightarrow 1$ sufficiently fast that (2.12) is satisfied (take $\alpha_{n}=1-1 / n^{2}$ for example). It then follows from Lemma 2.5 that $\mathscr{R}_{2}$ has the same boundary as $\mathscr{R}_{1}$.

THEOREM 2.6. Let $(X, \sigma, G)$ be an approximately transitive $G$-space. Then there exists a group-invariant Markov random walk $\mathscr{R}$ on $G$ with Poisson boundary $(B, \mu, G)$, and a G-equivariant isometry $\theta: L^{\infty}(X, \sigma)$ $\rightarrow L^{\infty}(B, \mu)$. I.e. an approximately transitive $G$-space is a factor of the Poisson boundary of some group-invariant Markov random walk on $G$.

Proof. Let $P(X, \sigma)$ be the space of finite measures $\nu$ on $X, \nu<\sigma$. We will construct a sequence of probability measures $\sigma_{n} \in P(X, \sigma)$ such that (i) $\overline{\mathrm{Co}}_{G}\left(\left(\sigma_{n}\right)_{n \in N}\right)=P(X, \sigma)$, and (ii) $\sigma_{n}=\lambda_{n} * \sigma_{n+1}$ where the $\lambda_{n}$ are probability measures on $G$. $\mathscr{R}$ will be the random walk defined by the $\lambda_{n}$.

Choose $\left(\nu_{n}\right)_{n \in N}$ dense in the $L^{1}$-norm in $P(X, \sigma)$. Choose $\varepsilon_{n}>0$, $\sum_{n \in N} \varepsilon_{n}<\infty$. Since $(X, \sigma, G)$ is approximately transitive, we can choose inductively probability measures $\sigma_{n}^{\prime} \in P(X, \sigma)$ such that

$$
d\left(\nu, \mathrm{Co}_{G} \sigma_{n}^{\prime}\right)<\varepsilon_{n} \quad \text { for } \nu=\sigma_{n-1}^{\prime}, \nu_{1}, \ldots, \nu_{n} .
$$

Then $\overline{\mathrm{Co}}_{G}\left(\left(\sigma_{n}^{\prime}\right)_{n \in N}\right)=P(X, \sigma)$, and there exist probability measures $\lambda_{n}$ on $G$ (see Remark 2.2 (ii) of [7]) such that

$$
\left\|\sigma_{n}^{\prime}-\lambda_{n} * \sigma_{n+1}^{\prime}\right\|<\varepsilon_{n+1} \text {. }
$$

For $n<m$ let

$$
\sigma_{n, m}=\lambda_{n} * \lambda_{n+1} * \cdots * \lambda_{m} * \sigma_{m+1}^{\prime} .
$$

For $n<m_{1}<m_{2}$ we have, using (2.22),

$$
\left\|\sigma_{n, m_{1}}-\sigma_{n, m_{2}}\right\|<\sum_{k=m_{1}}^{m_{2}} \varepsilon_{k+1}
$$


which tends to 0 as $m_{1}, m_{2} \rightarrow \infty$. Since $P(X, \sigma)$ is complete in the $L^{1}$-norm, we can define

$$
\sigma_{n}=\lim _{m \rightarrow \infty} \sigma_{n, m}
$$

Since $\|\lambda * \nu\| \leq\|\lambda\|\|\nu\|$ and $\sigma_{n, m}=\lambda_{n} * \sigma_{n+1, m}$ it follows that

$$
\sigma_{n}=\lambda_{n} * \sigma_{n+1} \text {. }
$$

Since

$$
\left\|\sigma_{n}-\sigma_{n}^{\prime}\right\|<\sum_{k=n+1}^{\infty} \varepsilon_{k} \underset{m \rightarrow \infty}{\rightarrow} 0
$$

it follows that the $\sigma_{n}$ also generate $P(X, \sigma)$.

Define $\Theta_{n}: L^{\infty}(X, \sigma) \rightarrow L^{\infty}(G, d g)$ by

$$
\left(\Theta_{n} f\right)(g)=\int f\left(g x^{-1}\right) d \sigma_{n}(x) .
$$

Using (2.25) we have

$$
\begin{aligned}
\left(\Theta_{n} f\right)(g) & =\iint f\left(g x^{-1}\right) d \lambda_{n}(k) d \sigma_{n+1}\left(k^{-1} x\right) \\
& =\iint f\left(g k y^{-1}\right) d \lambda_{n}(k) d \sigma_{n+1}(y) \\
& =\int\left(\Theta_{n+1} f\right)\left(g k^{-1}\right) d \lambda_{n}(k)=\Theta_{n+1} f * \tilde{\lambda}_{n} .
\end{aligned}
$$

Thus $\left(\Theta_{n} f\right)_{n \in N}$ is harmonic for the random walk $\mathscr{R}$. The map $\Theta$ is now defined by $\Theta: f \rightarrow\left(\Theta_{n} f\right)_{n \in N}$.

Using (2.1) and $(h f)(x)=f\left(h^{-1} x\right)$ we have

$$
\begin{aligned}
\left(\Theta_{n}(h f)\right)(g) & =\int f\left(h^{-1} g x\right) d \sigma_{n}(x) \\
& =\left(\Theta_{n} f\right)\left(h^{-1} g\right)=\left(h\left(\Theta_{n} f\right)\right)(g)
\end{aligned}
$$

which proves the $G$-equivariance of $\Theta$.

It remains to prove that $\Theta$ is an isometry. Since the $\sigma_{n}$ are probability measures, it follows from (2.26) that $\left\|\Theta_{n}\right\| \leq 1$, and hence $\|\Theta\| \leq 1$. We will prove that $\lim _{n \rightarrow \infty}\left\|\Theta_{n} f\right\|_{\infty}=\|f\|_{\infty}$ for all $f \in L^{\infty}(X, \sigma)$. Let $f \in L^{\infty}(X, \sigma), \varepsilon>0$. Then there exists $c$ with $|c|=\|f\|_{\infty}$ such that the set

$$
A=\left\{x \in X:|f(x)-c|<\frac{1}{2} \varepsilon\right\}
$$

satisfies $\sigma(A)>0$. Let $\sigma_{A}$ denote the restriction of $\sigma$ to $A$. By a theorem of Varadarajan ([16], Theorem 2.1.19), we can assume that the action of $G$ on $X$ is continuous. Since the $\sigma_{n}$ generate $P(X, \sigma)$ it 
follows (compare [7], Remark 2.2 (ii), (v)) that there exist $n<\infty$ and $\lambda \in L_{+}^{1}(G, d g),\|\lambda\|=\left\|\sigma_{A}\right\|=\sigma(A)$, such that

$$
\left\|\sigma_{A}-\int d g \lambda(g) g \sigma_{n}\right\|<\frac{1}{2} \varepsilon \sigma(A) /\|f\|_{\infty} .
$$

Let

$$
J=\int d g \lambda(g) \int d \sigma_{n}\left(g^{-1} x\right) f(x) .
$$

If follows from (2.30) that

$$
\left|J-\int f(x) d \sigma_{A}(x)\right|<\frac{1}{2} \varepsilon \sigma(A) .
$$

(2.29) implies that

$$
\left|c \sigma(A)-\int f(x) d \sigma_{A}(x)\right|<\frac{1}{2} \varepsilon
$$

Since $|c|=\|f\|_{\infty},(2.31)$ and (2.32) give

$$
|| J\left|-\|f\|_{\infty} \sigma(A)\right|<\varepsilon .
$$

But

$$
\begin{aligned}
J & =\int d g \lambda(g) \int d \sigma_{n}(y) f\left(g y^{-1}\right) \\
& =\int d g \lambda(g)\left(\Theta_{n} f\right)(g)
\end{aligned}
$$

and hence

$$
|J| \leq\left\|\Theta_{n} f\right\|_{\infty}\|\lambda\|=\left\|\Theta_{n} f\right\|_{\infty} \sigma(A) .
$$

(2.33) and (2.34) give

$$
\left\|\Theta_{n} f\right\|_{\infty}>\|f\|_{\infty}-\varepsilon
$$

and hence $\lim _{n \rightarrow \infty}\left\|\Theta_{n} f\right\|_{\infty}=\|f\|_{\infty}$.

For the last remark, recall that a factor of a $G$-space $(B, \mathscr{F}, \mu, G)$ is just the restriction of $(B, \mu, G)$ to a $\sigma$-algebra $\mathscr{F}_{0} \subset \mathscr{F}$ which is $G$-invariant. Here one takes $\mathscr{F}_{0}$ to be the $\sigma$-algebra generated by $\Theta\left(L^{\infty}(X, \sigma)\right)$.

\section{Eigenvalue lists and random walks.}

(i) Random walks on $R$. 
THEOREM 3.1. Let $\left(\lambda_{n 1}, \ldots, \lambda_{n k_{n}}\right)_{n \in N}$ be an eigenvalue list. There is naturally associated to this eigenvalue list a group-invariant Markov random walk on $R$ whose Poisson boundary is canonically identified, as an ergodic $R$-action, with the flow of weights for the ITPFI factor defined by this eigenvalue list.

THEOREM 3.2. The Poisson boundaries of the group-invariant Markov random walks on $R$ are precisely the approximately transitive $R$-actions.

Proof of Theorem 3.1. Let $A_{n}=\left\{1, \ldots, k_{n}\right\}$ and let $\mu_{n}$ be the probability measure on $A_{n}$ defined by $\mu_{n}(\{j\})=\lambda_{n j}$. Define $\Psi_{n}: A_{n} \rightarrow R$ by $\Psi_{n}(j)=\log \lambda_{n j}$, and let $\sigma_{n}=\Psi_{n}\left(\mu_{n}\right)$. The measures $\sigma_{n}$ define the desired random walk.

The flow of weights for $M$ can be constructed as follows ([6], Corollary 6.3). Let $(A, \mu)=\Pi_{n=1}^{\infty}\left(A_{n}, \mu_{n}\right)$. Let $R$ act on $A \times R$ by $(a, t) s=$ $(a, s+t)$. Define an equivalence relation $S$ on $A \times R$ by $(a, t) \sim$ $(b, u)(S)$ if there exists $m<\infty$ such that $a_{n}=b_{n}$ for all $n>m$ and $u-t=\sum_{n=1}^{m} \log \left(\lambda_{n b_{n}} / \lambda_{n a_{n}}\right)$. The flow of weights for $M$ is then the ergodic action of $R$ on the algebra $\mathscr{A}$ of $S$-invariant elements of $L^{\infty}(A \times R, \mu \times d t)$ where $d t$ is Lebesgue measure.

The Poisson boundary of the random walk was defined in $\S 2$ in terms of the asymptotic algebra $\mathscr{A}_{\Omega}$, which is the $S_{\Omega}$-invariant subset of $L^{\infty}\left(R \times R^{N}, d t \times \mu\right)$ where $S_{\Omega}$ is tail-equivalence for the Markov chain $Y_{n}=Y_{0}+\sum_{k=1}^{n} X_{k}$. Since $Y_{n}-Y_{n-1}=X_{n}$, it follows that in $R \times R^{N}$ we have $c \sim d\left(S_{\Omega}\right)$ if and only if there exists $m<\infty$ such that $c_{n}=d_{n}$ for all $n>m$ and $\sum_{n=0}^{m} c_{n}=\sum_{n=0}^{m} d_{n}$. Define $\Psi: A \times R \rightarrow R \times R^{N}$ by $\Psi\left(\left(a_{n}\right), t\right)=\left(t,\left(\Psi_{n}\left(a_{n}\right)\right)\right)$. Rewriting the above equation as $d_{0}-c_{0}=\sum_{n=1}^{m}\left(c_{n}-d_{n}\right)$, we see that the measure-space isomorphism $\Psi$ maps $\mathscr{A}$ to $\mathscr{A}_{\Omega}$. Finally, we note that $\Psi$ commutes with the $R$-actions when restricted to these $\sigma$-algebras.

Proof of Theorem 3.2. This follows immediately from Theorems 2.2 and 3.1, and the fact that the flows corresponding to the ITPFI factors are precisely the approximately transitive flows ([7], Theorem 8.3).

(ii) Random walks on $Z$. Let $0<T<\infty$. Let $M=\bigotimes_{n=1}^{\infty}\left(M_{n}, \varphi_{n}\right)$ be an ITPFI factor with $T \in T(M)$. Using either ([1], Lemma 11.2) or ([3], Theorem 1.3.2) one can choose the states $\varphi_{n}$ so that $\sigma_{T}^{\varphi_{n}}=1$ for all $n$. The eigenvalue list $\left(\lambda_{n 1}, \ldots, \lambda_{n k_{n}}\right)$ defined by the states $\varphi_{n}$ then satisfies $\left(\lambda_{n j} / \lambda_{n k}\right)^{i T}=1$ for all $n, j, k$ (i.e. the ratios $\lambda_{n j} / \lambda_{n k}$ are all 
some integer power of $\lambda=e^{-2 \pi / T}$ ). Hence we can write $\lambda_{n j}=\lambda^{q_{n}} D_{n}$, $q_{n j} \in Z$ where $D_{n}=\sum_{j=1}^{k_{n}} \lambda^{q_{n j}}$. Conversely any such eigenvalue list defines an ITPFI factor $M$ with $T \in T(M)$. Since $T(M)$ is the point spectrum of the flow of weights $F_{t}^{M}$, the flow can be built under the ceiling function of constant height $2 \pi / T$ with the base transformation $B=F_{2 \pi / T}^{M}$. The conjugacy class of $B$ determines the conjugacy class of the flow, and hence $M$ (up to isomorphism). We shall call $B$ the modular transformation.

Theorem 3.3. Let $0<T<\infty$. Let $\left(\lambda_{n 1}, \ldots, \lambda_{n k_{n}}\right)_{n \in N}$ be an eigenvalue list satisfying $\left(\lambda_{n j} / \lambda_{n k}\right)^{i T}=1$ for all $n, j, k$. This eigenvalue list defines an ITPFI factor $M$ with $T \in T(M)$, and all such factors are obtained in this way. There is naturally associated to this eigenvalue list a group-invariant Markov random walk on $Z$ whose Poisson boundary is canonically identified, as an ergodic Z-action, with the modular transformation $F_{2 \pi / T}^{M}$.

Theorem 3.4. The Poisson boundaries of the group-invariant Markov random walks on $Z$ are precisely the approximately-transitive Z-actions.

Proof of Theorem 3.3. $B=F_{2 \pi / T}^{M}$ can be constructed as follows. Let $(A, \mu)=\Pi_{n=1}^{\infty}\left(A_{n}, \mu_{n}\right)$ where $A_{n}=\left\{1, \ldots, k_{n}\right\}$ and $\mu_{n}(\{j\})=\lambda_{n j}$. Let $Z$ act on $A \times Z$ by $\alpha_{n}((a, p))=(a, p+n)$. Define an equivalence relation $S$ on $A \times Z$ by $(a, p) \sim(b, n)(S)$ if there exists $m<\infty$ such that $a_{n}=b_{n}$ for all $n>m$ and $r-p=\sum_{n=1}^{m}\left(q_{n b_{n}}-q_{n a_{n}}\right)$, where we have written $\lambda_{n j}=\lambda^{q_{n j}} D_{n}, \lambda=e^{-2 \pi / T}$ (see above). The modular transformation $B$ is then the restriction of $\alpha_{1}$ to the algebra $\mathscr{A}$ of $S$ invariant elements of $L^{\infty}(A \times Z, \mu \times \nu)$ where $\nu$ is any measure whose support is $Z$.

Define $\Psi_{n}: A_{n} \rightarrow Z$ by $\Psi_{n}(j)=q_{n j}$, and let $\sigma_{n}=\Psi_{n}\left(\mu_{n}\right)$. The measures $\sigma_{n}$ define the group-invariant Markov random walk on $Z$ associated to the given eigenvalue list. As above, the map $\Psi: A \times Z \rightarrow$ $Z \times Z^{N}$ given by $\Psi\left(\left(a_{n}\right), p\right)=\left(p,\left(\psi_{n}\left(a_{n}\right)\right)\right)$ identifies the algebra $\mathscr{A}$ with the asymptotic algebra $\mathscr{A}_{\Omega}$ of the random walk, and commutes with the restriction of the $Z$-action to these algebras.

Proof of Theorem 3.4. This follows immediately from Theorems 2.2 and 3.3, and the fact that all approximately transitive transformations occur as the modular transformation $F_{2 \pi / T}^{M}$ for some ITPFI factor $M$ with $T \in T(M)$ ([7], Theorem 8.3 and Lemma 2.5). 
(iii) Matrix-valued random walks on $R$ and $Z$. Let $M$ be an approximately type I (not necessarily ITPFI) factor. Let $\varphi$ be a faithful normal state on $M$. By [4] and [5] there exists an increasing sequence of conditional expectations $E_{n}$ on $M$ such that

(i) $E_{n} M$ is finite-dimensional,

(ii) $E_{n} E_{m}=E_{m} E_{n}=E_{n}$ if $n<m$,

(iii) $\varphi \circ E_{n}=\varphi$, and

(iv) strong $\lim _{n \rightarrow \infty} E_{n} x=x$ for all $x \in M$.

We will associate a "matrix-valued" random walk on $R$ to the triple $\left(M, \varphi,\left(E_{n}\right)\right)$.

Let $E_{n} M=\bigoplus_{j=1}^{l_{n}} M_{n j}$ be the central decomposition of $E_{n} M$ into finite type I factors $M_{n j}$. (The random walk at the $n$th step will take place in $l_{n}$ copies of $R$.) Consider the embedding of $E_{n} M$ in $E_{n+1} M$ relative to the state $\varphi$. Each $M_{n j}$ will appear $q_{n j l}$ times in $M_{n+1, l}$. Let $e_{n j}$ be the identity in $M_{n j}$, and let $e_{n j l p}$ be the $p$ th copy of $e_{n j}$ in $M_{n+1, l}$, $p=1, \ldots, q_{n j l}$. Then $e_{n j}=\sum_{l p} e_{n j l p}$. Let $\lambda_{n j l p}=\varphi\left(e_{n j l p}\right) / \varphi\left(e_{n j}\right)$. Then $\sum_{l p} \lambda_{n j l p}=1$ for all $n, j$.

For each $n \in N$, let $\sigma_{n j l}, j=1, \ldots, l_{n}, l=1, \ldots, l_{n+1}$ be the matrix of measures on $R$ obtained by assigning the weight $\lambda_{n j l p}$ to the point $\log \lambda_{n j l p}$ for each $p=1, \ldots, q_{n j l}$. Then $\sum_{l} \sigma_{n j l}(R)=1$ for all $n, j$. The random walk is now defined by giving the transition probability from the point $x$ in the $j$ th copy of $R$ at the $n$th step, to the point $y$ in the $l$ th copy of $R$ at the $(n+1) t h$ step, to be

$$
P_{n}((x, j),(y, l))=\sigma_{n j l}(\{y-x\}) .
$$

It is again possible to identify the flow of weights for $M$ with the Poisson boundary of the random walk. Recall that all ergodic flows occur as the flow of weights for some approximately type I factor [12]. Thus we get

THEOREM 3.5. Let $M$ be an approximately type I factor, $\varphi$ a faithful normal state on $M$, and $E_{n}$ an increasing sequence of conditional expectations as above. There is associated to this situation a "matrix-valued" group-invariant Markov random walk on $R$ whose Poisson boundary is identified, as an R-action, with the flow of weights for $M$. All ergodic flows occur as the Poisson boundaries of such random walks.

It would be more natural to phrase Theorem 3.5 in terms of the numbers $\lambda_{n j l p}$ and $\left.\varphi\right|_{E_{1} M}$. They determine a state $\varphi$ on the AF algebra $A$ which is the $C^{*}$-inductive limit of the $E_{n} M$. In general, such a state 
will not be a factor state. If this "generalized eigenvalue list" satisfies the factor condition, then $\pi_{\varphi}(A)$ " is an approximately type I factor. However, this is a somewhat more involved situation to describe.

As in section (ii) above, the corresponding "matrix-valued" random walks on $Z$ are obtained by considering a faithful normal state $\varphi$ on an approximately type $I$ factor $M$, satisfying $\sigma_{T}^{\varphi}=1$ for some $T>0$. We omit the details.

4. Application to the $T$ set. We will derive the $T$-set condition for eigenvalue lists ([3], Corollaire 1.3.9) by an extremely simple argument. We consider the problem in the more general setting of a group-invariant Markov random walk $\mathscr{R}$ on a locally compact abelian group $G$.

Definition 4.1. The $T$-set $T(\mathscr{R})$ is the set of all $\chi \in \hat{G}$ for which there exist $f^{\chi} \in L^{\infty}(B, \mu)$ (where $(B, \mu)$ is the Poisson boundary of $\mathscr{R}$ such that $\left(g f^{\chi}\right)(b)=\chi(g) f^{\chi}(b)$ for all $g \in G$, and a.e. $b \in B$.

THEOREM 4.2. Let $\mathscr{R}$ be a group-invariant Markov random walk on the locally compact abelian group $G$, given by the measures $\sigma_{n}$. Then $\chi \in T(\mathscr{R})$ if and only if

$$
\prod_{n=1}^{\infty}\left|\int \chi(g) d \sigma_{n}(g)\right|>0 .
$$

REMARK. For an ITPFI factor $M$ with the eigenvalue list $\left(\lambda_{n 1}, \ldots\right.$, $\lambda_{n k_{n}}$ ), let $\mathscr{R}$ be the associated random walk on $G=R$ (see Theorem 3.1). Then $T \in T(M)$ if and only if the character $\chi_{T}(x)=e^{i T x}$ is in $T(\mathscr{R})$. (4.1) then becomes

$$
\prod_{n=1}^{\infty}\left|\sum_{j=1}^{k_{n}} \lambda_{n j}^{1+i T}\right|>0,
$$

which is the desired condition.

Proof. Recall that the Poisson formula maps $f \in L^{\infty}(B, \mu)$ to bounded harmonic functions, and commutes with the $G$-action. Thus $\chi \in T(\mathscr{R})$ if and only if there exists a bounded harmonic function $h^{\chi}$ satisfying $\left(g h_{n}^{\chi}\right)(x)=\chi(g) h_{n}^{\chi}(x)$ for all $g, x \in G$, and all $n \in N$. In particular this gives $h_{n+1}^{\chi}(g)=\chi(g) h_{n+1}^{\chi}(e)$. Since $h_{n}=h_{n+1} * \tilde{\sigma}_{n+1}$, we get

$$
h_{n}^{\chi}(e)=\int h_{n+1}^{\chi}(g) d \sigma_{n+1}(g)=h_{n+1}^{\chi}(e) \int \chi(g) d \sigma_{n+1}(g) .
$$


Clearly such a bounded $h^{\chi}$ will exist if and only if (4.1) is satisfied.

If $G$ is not abelian, the question becomes which irreducible unitary representations $\Pi$ of $G$ occur. That is, when there is a subspace $V$ of the space of harmonic functions, so that for $h \in V$ we have $g h=$ $\Pi(g) h$ for some $\Pi$. The answer to this question is more involved.

APPENDIX A. Cocycles, the Mackey range, and the Poisson boundary of a group-invariant Markov random walk. Let $S$ be a standard Borel space, let $\mu$ be a probability measure on $S$, and let $K, G$ be groups. We suppose there is a right Borel action of $K$ on $S$ such that $\mu$ is quasi-invariant and ergodic under $K$. A Borel function $\alpha: S \times K \rightarrow G$ is called a cocycle if for all $k_{1}, k_{2} \in K, \alpha\left(s, k_{1} k_{2}\right)=\alpha\left(s, k_{1}\right) \alpha\left(s k_{1}, k_{2}\right)$. That is, $\alpha$ is a homomorphism from $S \times K$ (considered as a groupoid) to $G$

Given a cocycle $\alpha$, there is a natural action of $K$ on $S \times G$ defined by $(s, g) k=(s k, g \alpha(s, k))$. We denote the space $S \times G$, together with this $K$ action, by $S \times{ }_{\alpha} G$. There is also a natural action of $G$ on $S \times{ }_{\alpha} G$ defined by $\left(s, g_{1}\right) g_{2}=\left(s, g_{2}^{-1} g_{1}\right)$, which commutes with the $K$-action. Let $\nu$ be a measure equivalent to Haar measure on $G$, and let $\mathscr{A}$ be the fixed points of $L^{\infty}\left(S \times_{\alpha} G, \mu \times \nu\right)$ under the $K$-action. Then $\mathscr{A}$ is an abelian von Neumann algebra, and $G$ acts by $*$-automorphisms on $\mathscr{A}$. It follows that we can write $\mathscr{A}=L^{\infty}(S, \sigma)$ where $(X, \sigma)$ is an essentially unique ergodic $G$-space.

Definition A.1. The $G$-space $(X, \sigma)$ is called the Mackey range of the cocycle $\alpha$.

Now let $\mathscr{R}$ be a right group-invariant Markov random walk on the group $G$, defined by the probability measures $\sigma_{n}$. Let $S=G^{N}$ and let $\mu=\prod_{n=1}^{\infty} \sigma_{n}$. For the measure $\nu$ on $G$, we take the initial distribution $\sigma_{0}$ (see $\S 2$ ). Let $K$ be the group of sequences $\left(g_{n}\right)_{n \in N}$ where $g_{n} \in G$ and $g_{n}=e$ for all but a finite number of indices $n$. We define a right action of $K$ on $S$ by $\left(s_{n}\right)\left(g_{n}\right)=\left(s_{n} g_{n}\right)$. Note that the equivalence relation on $S$ defined by this $K$-action is just tail-equivalence. Given $\left(g_{n}\right) \in K$, there exists $m<\infty$ such that $g_{n}=e$ for all $n>m$. The equation

$$
\alpha\left(\left(s_{n}\right),\left(g_{n}\right)\right)=s_{1} s_{2} \cdots s_{m}\left(s_{m} g_{m}\right)^{-1} \cdots\left(s_{2} g_{2}\right)^{-1}\left(s_{1} g_{1}\right)^{-1}
$$

defines a cocycle $\alpha$, which can be thought of as the formal product $\left(\prod_{n=1}^{\infty} s_{n}\right)\left(\prod_{n=1}^{\infty} s_{n} g_{n}\right)^{-1}$. 
If $(s, g),\left(s^{\prime}, g^{\prime}\right) \in S \times_{\alpha} G$ belong to the same $K$-orbit, then there exists some $k \in K$ such that $s^{\prime}=s k$ and $g^{\prime}=g \alpha(s, k)$. Choose $m<\infty$ so that $k_{n}=e$ for all $n>m$. Then we have $s_{n}^{\prime}=s_{n}$ for all $n>m$ and

$$
g^{\prime}=g s_{1} s_{2} \cdots s_{m}\left(s_{m} g_{m}\right)^{-1} \cdots\left(s_{1} g_{1}\right)^{-1} \text {. }
$$

We now proceed to show that the fixed-point algebra $\mathscr{A}$ coincides with the asymptotic algebra $\mathscr{A}_{\Omega}$ defined in $\S 2$. It follows from this definition that $\mathscr{A}_{\Omega}$ is the $S_{\Omega}$-invariant subset of $L^{\infty}\left(S \times_{\alpha} G, \mu \times \sigma_{0}\right)$ where $S_{\Omega}$ is tail-equivalence for the Markov chain $\left(Y_{n}\right)$ where $Y_{n}=$ $Y_{0} X_{1} X_{2} \cdots X_{n}$. Since $Y_{n}=T_{n-1} X_{n}$, it follows that in $G \times G^{N}$ we have $(g, s) \sim\left(g^{\prime}, s^{\prime}\right)\left(S_{\Omega}\right)$ if and only if there exists $m<\infty$ such that $s_{n}=s_{n}^{\prime}$ for all $n>m$ and

$$
g s_{1} s_{2} \cdots s_{m}=g^{\prime} s_{1}^{\prime} s_{2}^{\prime} \cdots s_{m}^{\prime}
$$

which agrees with (A.2), where one makes the obvious identification $s_{n}^{\prime}=s_{n} g_{n}$. Thus $S_{\Omega}$-equivalence coincides with the equivalence relation defined by the $K$-action on $S \times{ }_{\alpha} G$. It follows that the Poisson boundary for the random walk $\mathscr{R}$ is the Mackey range of the cocycle $\alpha$.

APPENDix B. The Choquet boundary. Let $X$ be the convex proper cone of all (not necessarily bounded) positive harmonic functions for the group-invariant Markov random walk defined by the measures $\sigma_{n}$. The representing measure in Choquet's integral representation theory is unique if and only if $X$ is a lattice in its own order ([2], vol. II, p. 201).

LEMMA B1. $X$ is a lattice in its own order.

Proof. Let $f, g \in X$. Consider

$$
h_{n}^{k}=\left(f_{n+k} \wedge g_{n+k}\right) * \tilde{\sigma}_{n+k} * \tilde{\sigma}_{n+k-1} * \cdots * \tilde{\sigma}_{n+1}
$$

where $f_{s} \wedge g_{s}$ denotes the usual point-wise infimum. Since

$$
f_{s} \wedge g_{s} \leq\left(f_{s+1} \wedge g_{s+1}\right) * \tilde{\sigma}_{s+1}
$$

the $h_{n}^{k}$ are monotone decreasing in $k$. Since $h_{n}^{k} \geq 0$, we can define

$$
(f \wedge g)_{n}=\lim _{k \rightarrow \infty} h_{n}^{k} \text {. }
$$

Since $h_{n}^{k+1}=h_{n+1}^{k} * \tilde{\sigma}_{n+1}$, it follows from the monotone convergence theorem that $(f \wedge g)_{n}=(f \wedge g)_{n+1} * \tilde{\sigma}_{n+1}$, i.e. $f \wedge g$ is harmonic. 
Similarly, $f \vee g$ is defined by

$$
(f \vee g)_{n}=\lim _{k \rightarrow \infty}\left(f_{n+k} \vee g_{n+k}\right) * \tilde{\sigma}_{n+k} * \tilde{\sigma}_{n+k-1} * \cdots * \tilde{\sigma}_{n+1}
$$

Since the series is monotone increasing and bounded above by $f+g$, it follows that $f \vee g$ exists and is harmonic.

\section{REFERENCES}

[1] H. Araki and E. J. Woods, A classification of factors, Publ. Res. Inst. Math. Sci., Ser. A., 4 (1968), 51-130.

[2] G. Choquet, Lectures in Analysis, Benjamin: New York, 1969.

[3] A. Connes, Une classification des facteurs de type III, Ann. Sci. E.N.S., 4ème Série, 6 (1973), 133-252.

[4] - Classification of injective factors, Ann. of Math., 104 (1976), 73-115.

[5] , On hyperfinite factors of type III and Krieger's factors, J. Funct. Anal., 18, 318-327.

[6] A. Connes and M. Takesaki, The flow of weights on factors of type III, Tohoku Math. J., 29 (1977), 473-575.

[7] A. Connes and E. J. Woods, Approximately transitive flows and ITPFI factors, Ergodic Theory Dynamical Systems, 5 (1985), 203-236.

[8] A. Connes, J. Feldman, and B. Weiss, An amenable equivalence relation is generated by a single transformation, Ergodic Theory Dynamical Systems, 1 (1981), 431-450.

[9] F. P. Greenleaf, Invariant Means on Topological Groups, Van Nostrand: New York, 1969.

[10] U. Haagerup, Connes' bicentralizer problem and uniqueness of the injective factor of type III $_{1}$, Acta Math., 158 (1987), 95-148.

[11] W. Krieger, On the infinite product construction of non-singular transformations of a measure space, Invent. Math., 15 (1972), 144-163.

[12] On ergodic flows and the isomorphism of factors, Math. Ann., 233 (1976), 19-70.

[13] F. J. Murray and J. von Neumann, Rings of operators IV, Ann. of Math., 44 (1943), 716-804.

[14] D. Revuz, Markov Chains, North-Holland: Amsterdam, 1975.

[15] R. J. Zimmer, Amenable group actions and an application to Poisson boundaries of random walks, J. Funct. Anal., 27 (1978), 350-372.

[16] _ Ergodic Theory and Semisimple Groups, Birkhäuser: Basel, 1984.

Received May 2, 1988.

IHES

35, Route De Chartres

91440 Bures-Sur-Yvette, France

AND

QUEEN'S UNIVERSITY

KINGSTON, ONTARIO

CANADA K7L 3 N6 



\section{PACIFIC JOURNAL OF MATHEMATICS EDITORS}

\author{
V. S. VARADARAJAN \\ (Managing Editor) \\ University of California \\ Los Angeles, CA 90024 \\ Herbert Clemens \\ University of Utah \\ Salt Lake City, UT 84112 \\ THOMAS ENRIGHT \\ University of California, San Diego \\ La Jolla, CA 92093
}

R. FINN

Stanford University

Stanford, CA 94305

HeRmann FlaschKa

University of Arizona

Tucson, AZ 85721

VAUGHAN F. R. Jones

University of California

Berkeley, CA 94720

STEVEN KeRCKHOFF

Stanford University

Stanford, CA 94305
ROBION KIRBY

University of California

Berkeley, CA 94720

C. C. MOORE

University of California

Berkeley, CA 94720

HAROLD STARK

University of California, San Diego

La Jolla, CA 92093

\section{ASSOCIATE EDITORS}
R. ARENS
E. F. BECKENBACH
B. H. NeUMANN
F. WolF
K. YoshidA (1906-1982)

\section{SUPPORTING INSTITUTIONS}

UNIVERSITY OF ARIZONA

UNIVERSITY OF BRITISH COLUMBIA

CALIFORNIA INSTITUTE OF TECHNOLOGY

UNIVERSITY OF CALIFORNIA

MONTANA STATE UNIVERSITY

UNIVERSITY OF NEVADA, RENO

NEW MEXICO STATE UNIVERSITY

OREGON STATE UNIVERSITY

\author{
UNIVERSITY OF OREGON \\ UNIVERSITY OF SOUTHERN CALIFORNIA \\ STANFORD UNIVERSITY \\ UNIVERSITY OF HAWAII \\ UNIVERSITY OF TOKYO \\ UNIVERSITY OF UTAH \\ WASHINGTON STATE UNIVERSITY \\ UNIVERSITY OF WASHINGTON
}

The Supporting Institutions listed above contribute to the cost of publication of this Journal, but they are not owners or publishers and have no responsibility for its content or policies.

Mathematical papers intended for publication in the Pacific Journal of Mathematics should be in typed form or offset-reproduced (not dittoed), double spaced with large margins. Please do not use built up fractions in the text of the manuscript. However, you may use them in the displayed equations. Underline Greek letters in red, German in green, and script in blue. The first paragraph must be capable of being used separately as a synopsis of the entire paper. In particular it should contain no bibliographic references. Please propose a heading for the odd numbered pages of less than 35 characters. Manuscripts, in triplicate, may be sent to any one of the editors. Please classify according to the scheme of Math. Reviews, Index to Vol. 39. Supply name and address of author to whom proofs should be sent. All other communications should be addressed to the managing editor, or Elaine Barth, University of California, Los Angeles, California 90024.

There are page-charges associated with articles appearing in the Pacific Journal of Mathematics. These charges are expected to be paid by the author's University, Government Agency or Company. If the author or authors do not have access to such Institutional support these charges are waived. Single authors will receive 50 free reprints; joint authors will receive a total of 100 free reprints. Additional copies may be obtained at cost in multiples of 50 .

The Pacific Journal of Mathematics is issued monthly as of January 1966. Regular subscription rate: $\$ 190.00$ a year (5 Vols., 10 issues). Special rate: $\$ 95.00$ a year to individual members of supporting institutions.

Subscriptions, orders for numbers issued in the last three calendar years, and changes of address should be sent to Pacific Journal of Mathematics, P.O. Box 969, Carmel Valley, CA 93924, U.S.A. Old back numbers obtainable from Kraus Periodicals Co., Route 100, Millwood, NY 10546.

The Pacific Journal of Mathematics at P.O. Box 969, Carmel Valley, CA 93924 (ISSN 0030-8730) publishes 5 volumes per year. Application to mail at Second-class postage rates is pending at Carmel Valley, California, and additional mailing offices. Postmaster: send address changes to Pacific Journal of Mathematics, P.O. Box 969, Carmel Valley, CA 93924.

\section{PUBLISHED BY PACIFIC JOURNAL OF MATHEMATICS, A NON-PROFIT CORPORATION}




\section{Pacific Journal of Mathematics}

Vol. 137, No. $2 \quad$ February, 1989

Alain Connes and E. J. Woods, Hyperfinite von Neumann algebras and Poisson boundaries of time dependent random walks ............ 225

R. Coquereaux and D. Kastler, Remarks on the differential envelopes of

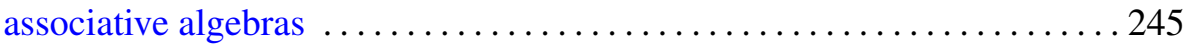

Uffe Haagerup, The injective factors of type $\mathrm{III}_{\lambda}, 0<\lambda<1 \ldots \ldots \ldots 265$

Vaughan Jones, On knot invariants related to some statistical mechanical

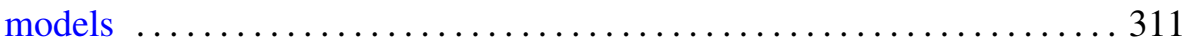

Paul Scott Muhly, Kichi-Suke Saito and Baruch Solel, Coordinates for triangular operator algebras. II ......................... 335

Klaus Schmidt, Mixing automorphisms of compact groups and a theorem by Kurt Mahler ........................................ 371

Irving E. Segal, Algebraic characterization of the vacuum for quantized

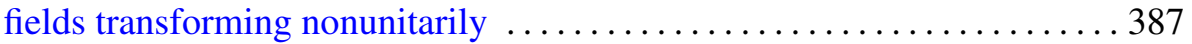

Colin Eric Sutherland and Masamichi Takesaki, Actions of discrete amenable groups on injective factors of type $\mathrm{III}_{\lambda}, \lambda \neq 1 \ldots \ldots \ldots \ldots 405$ 